\title{
Sistema Integrado de Análise e Visualização Epidemiológica Georreferenciada Online para Telemedicina
}

\author{
Andrei de Souza Inácio ${ }^{1,2}$, Alexandre Savaris ${ }^{2}$, João Marcus Alves ${ }^{1,2}$, Aldo von Wangenheim ${ }^{1,2}$ \\ ${ }^{1}$ Programa de Pós-Graduação em Ciência da Computação (PPGCC) \\ Universidade Federal de Santa Catarina (UFSC) \\ Florianópolis, SC - Brasil \\ ${ }^{2}$ Laboratório de Telemedicina \\ Instituto Nacional para Convergência Digital (INCoD) \\ Florianópolis, SC - Brazil \\ \{andrei, savaris, joao\}@telemedicina.ufsc.br, aldo.vw@ufsc.br
}

\begin{abstract}
This paper presents a georeferenced epidemiological analysis tool that is integrated into the infrastructure of the Santa Catarina State Integrated Telemedicine and Telehealth System (STT/SC). GISTelemed integrates STT/SC with the Geographic Information System of Santa Catarina (SIG@SC) and supports recovery, georeferenced visualization and epidemiological analysis on structured and semi-structured data. Results from a case study indicate a positive perception of the usefulness of GISTelemed by experts and health managers.
\end{abstract}

Resumo. Este trabalho apresenta o GISTelemed, uma ferramenta de análise epidemiológica georreferenciada que é integrada à infraestrutura do Sistema Catarinense Integrado de Telemedicina e Telessaúde (STT/SC). O GISTelemed integra o STT/SC com o Sistema de Informações Geográficas de Santa Catarina (SIG@SC) e permite a recuperação, visualização georreferenciada e análise epidemiológica em dados estruturados e semiestruturados. Resultados obtidos através de um estudo de caso indicam uma percepção positiva em relação à utilidade do GISTelemed por especialistas e gestores de saúde.

\section{Introdução}

Avanços na análise de dados relativos à mortalidade e sobrevivência populacional têm permitido aos pesquisadores examinar outras dimensões da saúde, medidas pela morbidade, incapacidade, acesso a serviços, qualidade de atendimento, condições de vida e fatores ambientais [Rede Interagencial de Informação para a Saúde 2008], contribuindo para uma observação mais eficaz do estado de saúde da população.

A epidemiologia (uma importante área da saúde pública [Friis and Sellers 2013]) se beneficia da fácil e rápida visualização de dados, pois esta auxilia os epidemiologistas a entenderem as relações entre os potenciais fatores de risco de doenças e suas morbidades e mortalidades associadas [Shi et al. 2007]. Como ferramenta de suporte para aquisição de dados, apesar de não ser muito considerada em situações epidemiológicas, a telemedicina oferece a capacidade de coletar e organizar os dados de pacientes, auxiliando a vigilância epidemiológica através da identificação e acompanhamento de questões de saúde pública [World Health Organization 2010]. O uso da telemedicina também permite o aprimoramento de investigações epidemiológicas, o controle de doenças e a gestão de casos 
clínicos [Ohannessian 2015], e eficientemente ajuda os gestores de políticas públicas a direcionar recursos de saúde e profissionais com base em cenários e relações concretas de morbidade [de Souza Inacio et al. 2014].

Um exemplo de sistema de telemedicina amplamente utilizado é o Sistema Catarinense Integrado de Telemedicina e Telessaúde (STT/SC). Desenvolvido no ano de 2005 através de uma parceria entre a Secretaria de Estado da Saúde de Santa Catarina (SES/SC) e a Universidade Federal de Santa Catarina (UFSC) [Maia et al. 2006], o STT/SC fornece serviços síncronos e assíncronos de telemedicina e telessaúde via web e dispositivos móveis [de Souza Inacio et al. 2014] que suportam radiologia, eletrocardiografia, dermatologia, análises clínicas e educação em saúde. Disponível em todos os municípios do Estado de Santa Catarina, o STT/SC armazena dados estruturados e semiestruturados de mais de quatro milhões de exames, demandando estratégias para a coleta, organização e visualização dos dados adquiridos por um grande número de profissionais (por exemplo, médicos, profissionais de cuidados primários, administradores de sistema, gestores de saúde) previamente cadastrados.

O georreferenciamento de dados, que consiste na associação de um dado à uma coordenada na superfície terrestre [Moreira et al. 2007], permite a análise da distribuição espacial de incidência de eventos e fornece informações importantes para compreensão, prevenção e monitoramento de doenças [Cavicchioli Neto et al. 2014]. Para o processamento desses dados são usados os Sistemas de Informações Geográficas (SIGs), um conjunto de sistemas de computador com capacidades de coletar, armazenar, gerenciar, analisar e apresentar informações georreferenciadas em mapas temáticos [de Pina and Santos 2010].

Neste trabalho apresentamos o GISTelemed, uma abordagem para a organização, recuperação e análise epidemiológica georreferenciada online dos dados armazenados no STT/SC. Focado em telecardiologia, teledermatologia e doenças infecciosas (hepatite B, HIV, toxoplasmose, dengue, vírus zika e febre chikungunya), o GISTelemed coleta dados estruturados e semiestruturados de diversas fontes disponíveis (Sistemas Gerenciadores de Banco de Dados Relacionais - SGBDRs e Sistemas de Comunicação e Arquivamento de Imagens - PACSs), empregando vocabulários controlados para a catalogação e indexação dos dados e provendo uma interface customizada para busca e visualização.

O restante deste trabalho está organizado como segue: a Seção 2 apresenta os trabalhos relacionados em sistemas de informações epidemiológicas; a Seção 3 descreve a arquitetura do GISTelemed e o modelo de qualidade customizado para sua avaliação. Os dados coletados por meio dos questionários de avaliação de qualidade são analisados e discutidos na Seção 4, orientando as conclusões sobre a viabilidade da abordagem de avaliação apresentadas na Seção 5.

\section{Trabalhos Relacionados}

Várias abordagens têm sido desenvolvidas com foco em análise de doenças infecciosas, tais como cólera, gripe H1N1 e leishmaniose [Rasam et al. 2011, Jiang et al. 2011, Jian-rong and Yan-jun 2010, Amoli 2011], usando um SIG como uma forma de melhorar a qualidade, acesso e uso das informações de saúde. As ferramentas baseadas em SIG podem ser úteis também para pesquisadores e gestores de políticas públicas de saúde, por permitir a exploração de mapas para entender a dinâmica espacial das doenças 
[Kandwal et al. 2009]. Com um enfoque diferente, outras abordagens concentram-se no monitoramento das condições de saúde e alerta de doenças através de dados coletados a partir de mídias e redes sociais [Freifeld et al. 2008, Widener and Li 2014, Parker et al. 2013], explorando técnicas de recuperação de informação e classificação como uma alternativa para identificação de doenças.

No contexto da saúde pública, o CDC Wonder ${ }^{1}$ e OASIS ${ }^{2}$ fornecem recursos de informação históricos para pesquisadores e profissionais de saúde pública, a fim de auxiliar o processo de tomada de decisão. A população em geral pode acessar informações detalhadas sobre saúde pública, especificando critérios de pesquisa e visualizando os resultados em tabelas ou mapas.

No contexto da telemedicina, algumas abordagens têm sido desenvolvidas com o intuito de coletar, processar e indexar dados estruturados e semiestruturados [Farruggia et al. 2014, Costa et al. 2009]. Essas soluções são integradas a sistemas PACS, permitindo a busca e recuperação de imagens e laudos de exames; contudo, não há integração com SIGs para visualização georreferenciada dos dados.

O GISTelemed (apresentado neste trabalho) difere das ferramentas acima mencionadas por integrar no mesmo sistema a capacidade de indexar dados estruturados (relacionais) e semiestruturados (laudos armazenados no padrão DICOM SR - Digital Imaging and Communications in Medicine - Structured Reports), buscar e recuperar informações a partir de pesquisas estrututuradas e em texto livre, e apresentar os resultados em tabelas, gráficos e mapas, todos integrados à uma aplicação de telemedicina (STT/SC).

\section{Materiais e Métodos}

$\mathrm{Na}$ infraestrutura do STT/SC, o GISTelemed é integrado como um módulo ao Sistema de Informações STT, usando tecnologias relacionadas à extração, indexação, busca, recuperação e visualização de dados. Através da interface do usuário, é possível avaliá-lo de acordo com a perspectiva de seus usuários finais em termos de qualidade percebida.

\subsection{A Arquitetura do GISTelemed}

O módulo GISTelemed, apresentado na Figura 1, estrutura-se em cinco componentes, distribuindo as tecnologias adotadas para atender aos processos ETL (Extract Transform Load), o componente motor de busca, as operações de pré-processamento e pósprocessamento e a interface do usuário. O processo ETL é responsável por acessar as fontes de dados estruturadas (bancos de dados relacionais) e semiestruturadas (PACSs), coletando dados para indexação. Processos individuais foram criados para atender às características específicas de cada conjunto de dados (exames de HIV, dengue, hepatite B, toxoplasmose, vírus Zika e febre Chikungunya, extraídos do sistema do Laboratório Central de Saúde Pública de Santa Catarina - LACEN/SC; laudos de exames de cardiologia e dermatologia extraídos do Sistema de Informações do STT e do Servidor DICOM - PACS). Esses processos são executados via Mirth ${ }^{\circledR}$ Connect $^{3}$ v.3.2.0 (uma ferramenta open-source para envio bi-direcional de mensagens por meio de diversos protocolos). Os

\footnotetext{
${ }^{1}$ http: //wonder.cdc.gov/

${ }^{2}$ https://oasis.state.ga.us/

${ }^{3}$ https://www.mirth.com/Products-and-Services/Mirth-Connect
} 


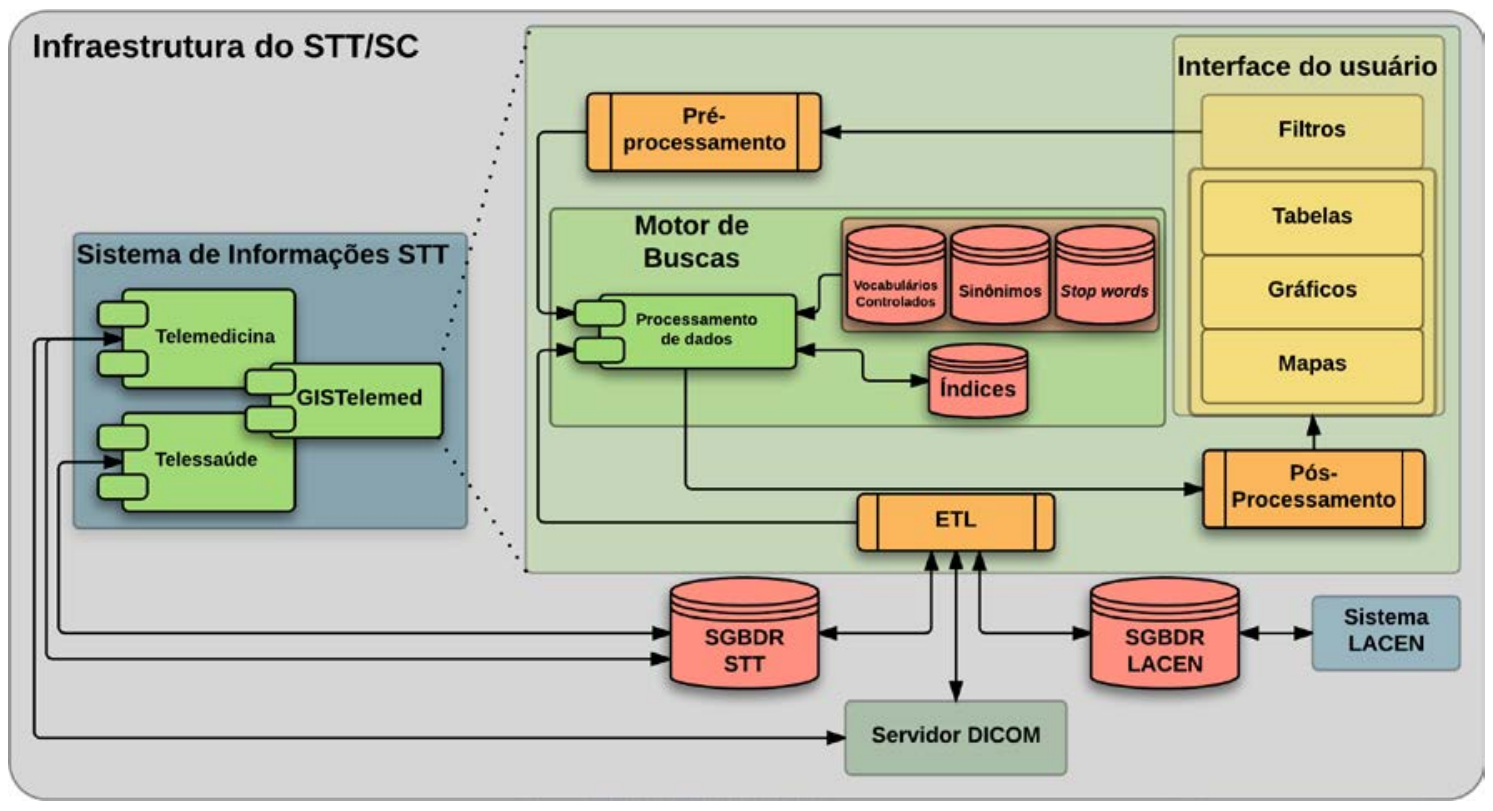

Figura 1. Arquitetura do módulo GISTelemed integrado ao STT/SC.

dados coletados são processados e convertidos em documentos XML (eXtensible Markup Language) e enviados via método HTTP POST (HyperText Transfer Protocol) para o componente motor de busca.

O componente motor de busca, baseado em Apache Solr ${ }^{4}$ v.4.10 (um servidor de indexação e buscas open-source), é responsável por receber e indexar os dados provenientes do processo ETL; ele responde, também, pela execução de buscas e recuperação de documentos de acordo com os termos presentes na pesquisa recebida via interface do usuário. Ambos os passos de indexação e recuperação empregam as técnicas de recuperação de informação stemming e stop words para melhorar o desempenho das consultas em texto livre.

As operações de pré-processamento e pós-processamento são responsáveis, respectivamente, por padronizar os filtros e os campos de filtro selecionados antes da submissão das instruções de pesquisa ao motor de busca, e pela normalização dos resultados que são apresentados em tabelas, gráficos e mapas.

A interface do usuário (apresentada na Figura 2) foi desenvolvida para permitir que usuários experientes e inexperientes possam realizar consultas e análises epidemiológicas. Através da interface de filtros (a) é possível selecionar os parâmetros de busca a partir de um conjunto pré-definido de filtros, bem como usar texto livre; a interface também permite escolher os dados que serão apresentados como resultado, usando um mecanismo de arrastar-e-soltar. Através da interface de resultados é possível visualizar o resultado das buscas em gráficos (b), tabelas (c), ou em três tipos de mapas (ocorrências (d), calor (e), e coroplético (f)) usando o formato GeoJSON ${ }^{5}$ e a biblioteca OpenLayers ${ }^{6}$.

O mapa do Estado de Santa Catarina é carregado usando o WMS (Web Map Ser-

\footnotetext{
${ }^{4}$ http: //lucene. apache.org/solr/

${ }^{5}$ http: //geojson.org/

${ }^{6}$ http: //openlayers.org/
} 


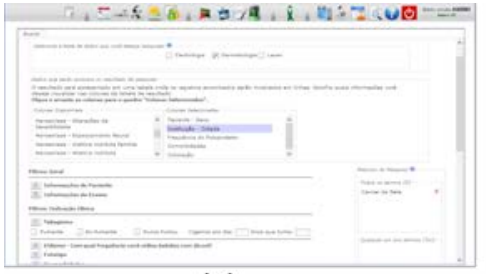

(a)

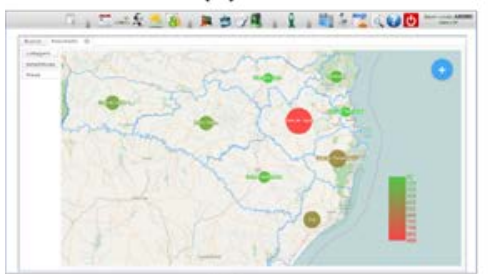

(d)

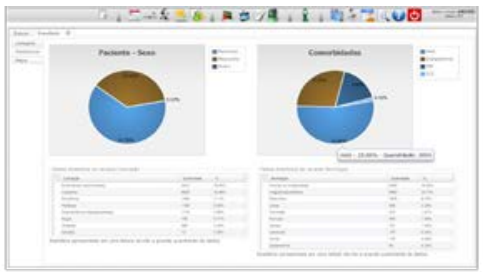

(b)

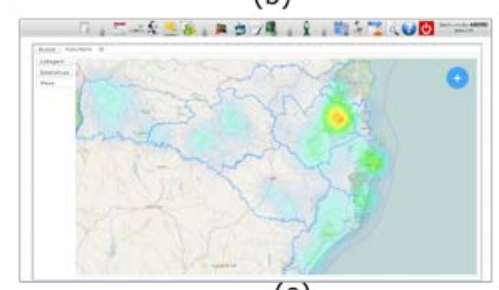

(e)

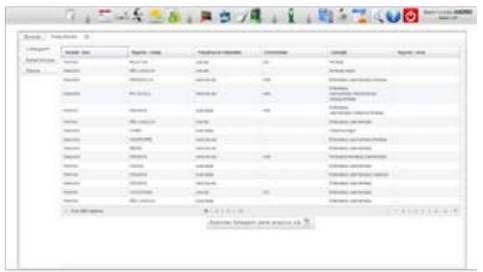

(c)

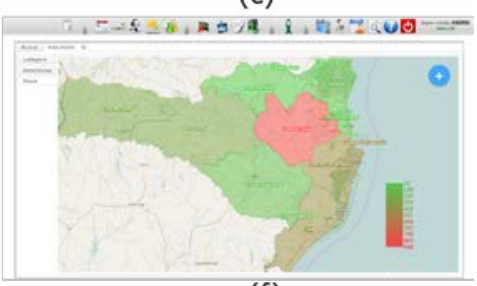

(f)

Figura 2. Interface do módulo GISTelemed - filtros (a), resultados em gráficos (b), tabelas (c), mapas: ocorrências (d), calor (e) e coroplético (f).

vice) disponibilizado pelo SIG@ $\mathrm{SC}^{7}$, um serviço desenvolvido através de uma parceria entre a UFSC e o Centro de Informática e Automação do Estado de Santa Catarina (CIASC) que oferece acesso público aos dados geográficos vetoriais e matriciais (raster). Os dados plotados em mapas podem ser visualizados por estabelecimento de saúde, município ou região, permitindo um acesso rápido à distribuição espacial de doenças específicas ou exames realizados.

\subsection{Estratégia de Validação}

Com o objetivo de avaliar a qualidade de software do módulo GISTelemed foi realizado um estudo de caso com os usuários finais do STT/SC (incluindo médicos, gestores de políticas de saúde pública e pesquisadores). Os participantes foram convidados a seguir um roteiro predefinido, utilizando o módulo GISTelemed para executar tarefas de pesquisa e recuperação de dados. A tarefa solicitada consiste na pesquisa do número de ocorrências de uma doença específica (de acordo com a especialidade do usuário), a análise estatística das variáveis idade, sexo e cidade do paciente, e a distribuição dos dados no mapa; sua avaliação foi realizada por meio de um questionário.

De um total de 37 usuários convidados, 26 aceitaram participar da avaliação (taxa de aceitação de 70,3\%). Concluída a tarefa, os usuários responderam ao questionário com a finalidade de colher dados sobre a sua percepção de qualidade relativa ao módulo GISTelemed. O questionário foi personalizado de acordo com o AdEQUATE (questionnAire for Evaluation of QUAlity in TElemedicine) [Alves et al. 2015], um modelo de avaliação para atender às especificidades e necessidades dos sistemas de telemedicina baseado na norma ISO/IEC 25010 [Iso.org 2011], no TAM (Technology Acceptance Model) [Davis 1989], e no SUS (System Usability Scale) [Brooke 1996]. O modelo decompõe conceitos abstratos de qualidade através dos fatores de qualidade da norma ISO/IEC 25010 com respeito à sua relevância para sistemas de telemedicina. Todos os itens do questionário foram formulados como afirmações, com o objetivo de eliminar erros de interpretação e reduzir a probabilidade de erros nas respostas [Sauro and Lewis 2011]; uma escala Likert de 4 pontos [Boone and Boone 2012] foi usada, incluindo as alterna-

\footnotetext{
${ }^{7}$ http://sigsc.sds.sc.gov.br/
} 
tivas "Não se aplica", "Não sei responder"e "Não entendi o item". As respostas aos questionários foram usadas para computar a distribuição de cada opção respondida para cada item de avaliação, usando uma abordagem semelhante ao SUS para sumarização dos dados.

\section{Resultados e Discussões}

Os dados adquiridos através do questionário foram analisados usando estatísticas descritivas, adotando a mediana como um representante para o meio da distribuição, e quartis para agrupamento [Boone and Boone 2012]. As pontuações das respostas dadas para cada item do questionário foram calculadas de maneira similar ao método usado pelo SUS [Finstad 2010], conforme fórmula apresentada em (1).

$$
S_{n}=\left(\sum_{i=1}^{26} W\left(\tilde{R}_{i}\right)\right) * \frac{100}{78}
$$

Em (1), $\tilde{R}_{i}$ corresponde à média das respostas obtidas no questionário para a subcaracterística $n$ pelo participante $i$, e $W\left(\tilde{R}_{i}\right)$ é uma função-peso com base em uma opção de resposta assumindo os seguintes valores: 3 para "Concordo totalmente", 2 para "Concordo", 1 para "Discordo" e 0 para "Discordo totalmente". As opções de respostas "Não sei responder", "Não se aplica" e "Não entendi o item" são ignoradas. O último componente em (1) garante que $S_{n}$ varie de 0 (pior grau de qualidade - todos os 26 participantes escolheram a opção "Discordo totalmente") até 100 (grau excepcional de qualidade todos os 26 participantes escolheram a opção "Concordo totalmente").

As pontuações de cada subcaracterística são apresentadas na Figura 3 (b), variando de 0 (pior grau de qualidade) até 100 (melhor grau de qualidade). Nós podemos observar que "Aprendizado", "Eficiência", "Conforto", "Utilidade"e "Corretude Funcional"foram as subcaracterísticas que receberam as melhores avaliações, indicando a boa percepção e aceitação do sistema do ponto de vista do usuário final. Algumas subcaracterísticas, principalmente as presentes no quartil inferior, receberam baixa pontuação, pois as opções de resposta "Não se aplica", "Não entendi o item"e "Não sei responder"não foram consideradas para o cálculo. Além disso, as subcaracterísticas com pontuação menor que 50 tiveram menos de $60 \%$ das respostas respondidas positivamente ou negativamente, conforme apresentado na Figura 3 (a). Nós também podemos observar na Figura 3 (b) que, de acordo com os usuários, as piores subcaracterísticas avaliadas foram "Acessibilidade", "Interoperabilidade", "Recuperabilidade"e "Tolerância a falhas". No entanto, uma vez que a média é maior que 50, pode-se notar uma percepção positiva da qualidade do GISTelemed. O intervalo interquartil (IQR) é de 16, demonstrando uma dispersão baixa.

A Figura 3 (a) apresenta a proporção das respostas de acordo com as subcaracterísticas do padrão ISO/IEC 25010. Nós podemos observar diferenças na posição de algumas subcaracterísticas quando comparadas com a Figura 3 (b); isso ocorre pois o gráfico apresentado na Figura 3 (a) apresenta a distribuição das respostas, e ele é apresentado em ordem decrescente de pontuação. "Eficiência", por exemplo, apesar de ter a mesma pontuação da subcaracterística "Aprendizado"calculada por (1), tem uma pior avaliação devido ao maior número de respostas negativas. Também é possível constatar que mais de $40 \%$ dos usuários responderam "Não se aplica"ou "Não sei responder"para as subcaracterísticas "Tolerância a falhas", "Recuperabilidade", "Interoperabilidade", e 


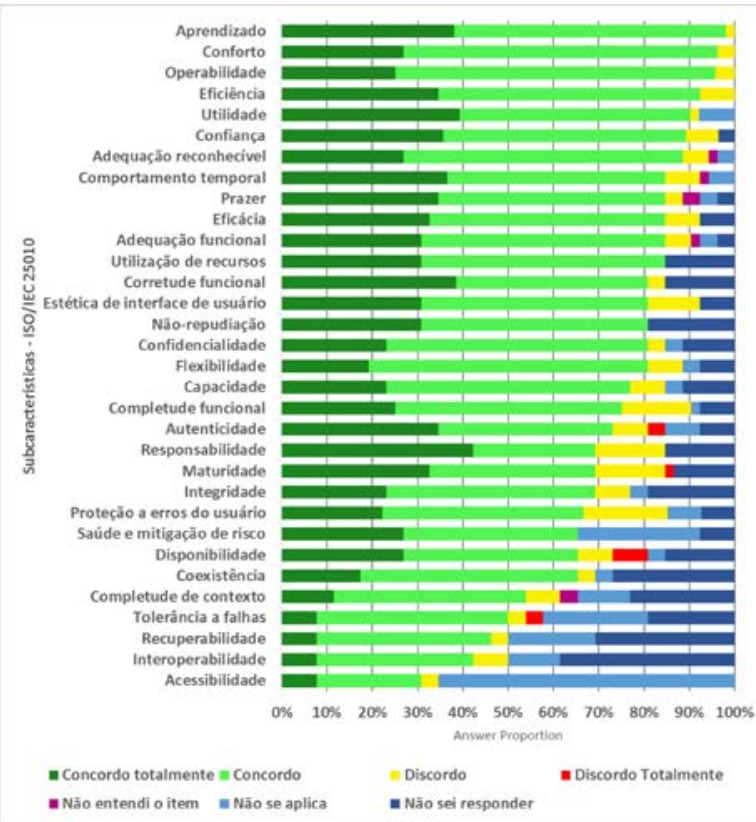

(a)

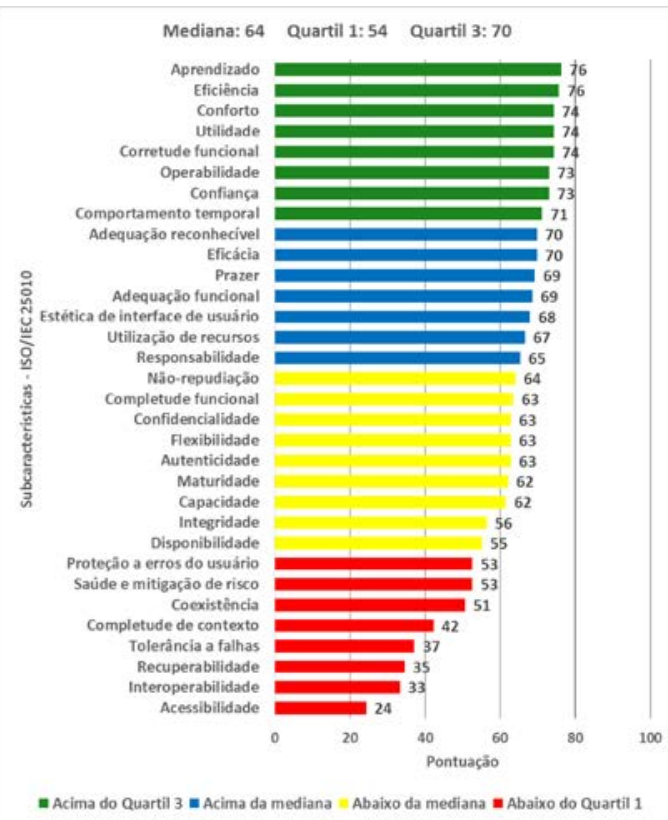

(b)

Figura 3. Resultados do estudo de caso de acordo com as subcaracterísticas de qualidade da norma ISO/IEC 25010 - (a) Proporção das respostas, (b) Pontuação da qualidade.

"Acessibilidade"; isso pode indicar que os usuários não conseguem avaliar o item através de um questionário, ou que os usuários não possuem experiência em usar o GISTelemed. "Acessibilidade"teve mais de 60\% das respostas como "Não se aplica", podendo indicar um problema no GISTelemed referente a essa subcaracterística, ou um viés de seleção dos participantes - de todos os participantes, nenhum possuía necessidades especiais.

A norma ISO/IEC 25010 define, também, duas dimensões: Qualidade em uso, associada com o grau com que o sistema está em conformidade com os objetivos dos usuários em suas atividades; e Qualidade do Produto, relacionada com as propriedades estáticas do software e as propriedades dinâmicas do sistema de computador [Iso.org 2011]. De acordo com a Figura 4, o GISTelemed foi avaliado positivamente por $80,31 \%$ dos participantes para a qualidade em uso - apenas $5,41 \%$ responderam negativamente, e 14,29\% responderam as opções "Não sei responder"e "Não se aplica". Em relação à qualidade do produto, o GISTelemed foi avaliado positivamente por 74,71\% dos participantes; apenas 7,34\% responderam negativamente, e 17,94\% responderam as opções "Não sei responder"e "Não se aplica". Considerando que a maioria dos itens

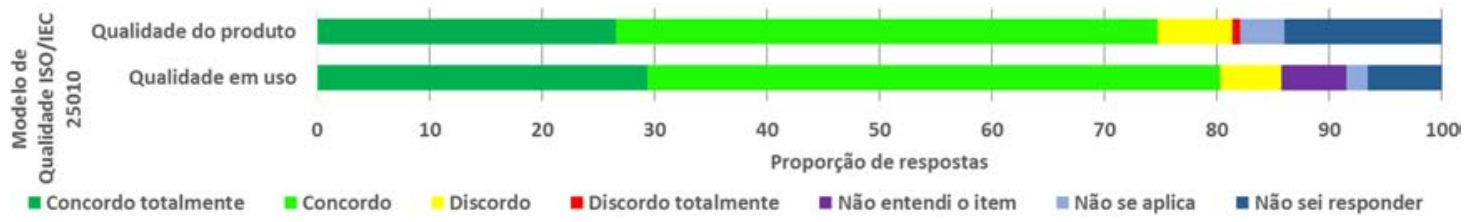

Figura 4. Proporção das respostas do modelo de qualidade ISO/IEC 25010.

foram avaliados positivamente, a mediana calculada para as subcaracterísticas foi 64 , e $84,4 \%$ das subcaracterísticas tiveram a pontuação maior que 50, nós podemos atestar a 
boa percepção da qualidade e aceitação do módulo GISTelemed pelos usuários finais do $\mathrm{STT} / \mathrm{SC}$.

\subsection{Ameaças à Validade}

Uma das ameaças relacionadas com a validade interna é o viés de seleção. Foram convidados apenas médicos pesquisadores, os gestores de políticas de saúde pública e epidemiologistas a responderem o questionário; isso é devido a razões práticas: GISTelemed foi desenvolvido principalmente como um módulo para análise epidemiológica, e estes são os seus potenciais utilizadores.

Uma possível ameaça relacionada ao viés de construção é o uso do modelo de avaliação AdEQUATE. O AdEQUATE não foi extensivamente validado por um grande número de usuários, e no contexto de outros sistemas; no entanto, acreditamos que esta ameaça é minimizada porque o questionário adotado teve seus itens sistematicamente derivados da norma ISO/IEC 25010, TAM e SUS, que são métodos largamente utilizados.

Outra ameaça identificada relacionada com um viés de seleção é que alguns entrevistados tiveram seu primeiro contato com GISTelemed durante o processo de avaliação, o que pode ter influenciado as respostas para alguns itens. Para minimizar esta ameaça, as opções "Não se aplica", "Não sei responder", e "Não entendi o item"foram incluídas como respostas válidas.

\section{Discussão e Conclusões}

Este trabalho apresenta o GISTelemed, uma ferramenta de análise epidemiológica georreferenciada integrada ao sistema de telemedicina STT/SC. Com o objetivo de ser usado por médicos, gestores de políticas de saúde pública e pesquisadores, o módulo tem como objetivo contribuir para o estudo das condições crônicas e infecciosas de doenças, em orientar a tomada de decisões de cuidados de saúde, e em contribuir para o desenvolvimento e avaliação das ações intervencionistas para controle e prevenção de doenças.

Os resultados do nosso estudo de caso utilizando o questionário AdEQUATE mostram que o GISTelemed tem uma boa avaliação em relação à qualidade do software percebida pelos usuários, de acordo com medidas que incluem "Eficácia", "Eficiência", "Satisfação", "Usabilidade", "Confiabilidade"e "Utilidade". Entretanto, alguns itens avaliados não tiveram um bom desempenho, como "Acessibilidade", "Interoperabilidade", "Recuperabilidade "e "Tolerância a falhas". Além disso, este trabalho mostra a viabilidade na avaliação de softwares de telemedicina por meio de questionários sistematicamente derivados e personalizados de acordo com o modelo de avaliação AdEQUATE.

A incorporação do georreferenciamento ao sistema, além de possibilitar a visualização dos dados em mapas, permitiu também analisar a evolução de surtos e ocorrências das doenças no tempo. Uma limitação do uso do SIG neste trabalho é a dificuldade de manter frequentemente a base de dados atualizada com as coordenadas dos estabelecimentos de saúde que registram as informações no STT/SC.

Como trabalhos futuros, buscaremos a inclusão de dados de Telessaúde (teleconsultorias), capacidades melhoradas de cruzamento de dados entre modalidades de exames e melhorias na interface do mapa para permitir análise temporal sobre os dados. 


\section{Agradecimentos}

Este trabalho teve o apoio da Secretaria de Estado da Saúde de Santa Catarina - SES/SC, do Conselho Nacional de Desenvolvimento Científico e Tecnológico - CNPq e da Fundação de Amparo à Pesquisa de Santa Catarina - FAPESC.

\section{Referências}

Alves, J. M., Wangenheim, C. G. v., Savaris, A., and Wangenheim, A. v. (2015). AdEQUATE Software QualityEvaluation Model v1.0. Brazilian Institute for Digital Convergence, 1 edition.

Amoli, G. M. (2011). Gis-based risk map analysis of leishmaniasis disease in isfahan, iran. In IEEE Symposium on Business, Engineering and Industrial Applications (ISBEIA 2011), pages 275-280.

Boone, H. N. and Boone, D. A. (2012). Analyzing likert data. Journal of Extension, 50:1 -5 .

Brooke, J. (1996). SUS: A quick and dirty usability scale. Usability evaluation in industry.

Cavicchioli Neto, V., Chiari, N. S., Pisa, I. T., and Alvez, D. (2014). Desenvolvimento e integração de mapas dinâmicos georreferenciados para o gerenciamento e vigilância em saúde. Journal of health informatics, 6(1):4.

Costa, C., Freitas, F., Pereira, M., Silva, A., and Oliveira, J. L. (2009). Indexing and retrieving dicom data in disperse and unstructured archives. International Journal of Computer Assisted Radiology and Surgery, 4(1):71-77.

Davis, F. D. (1989). Perceived usefulness, perceived ease of use, and user acceptance of information technology. MIS Quarterly, 13(3):319-340.

de Pina, M. F. and Santos, S. M. (2010). Conceitos Básicos de Sistemas de Informação Geográfica e Cartografia Aplicados à Saúde. OPAS Ministério da Saúde.

de Souza Inacio, A., de Macedo, D. D. J., Andrade, R., and von Wangenheim, A. (2014). Designing an information retrieval system for the stt/sc. In IEEE 16th International Conference on e-Health Networking, Applications and Services (Healthcom 2014), pages 500-505.

Farruggia, A., Magro, R., and Vitabile, S. (2014). A text based indexing system for mammographic image retrieval and classification. Future Generation Computer Systems, 37(0):243-251.

Finstad, K. (2010). The usability metric for user experience. Interacting with Computers, 22(5):323-327.

Freifeld, C. C., Mandl, K. D., Reis, B. Y., and Brownstein, J. S. (2008). Healthmap: Global infectious disease monitoring through automated classification and visualization of internet media reports. Journal of the American Medical Informatics Association: JAMIA, 15(2):150-157.

Friis, R. H. and Sellers, T. (2013). Epidemiology for public health practice. Jones \& Bartlett Learning. 
Iso.org (2011). Iso/iec 25010:2011 - systems and software engineering - systems and software quality requirements and evaluation (square) - system and software quality models.

Jian-rong, C. and Yan-jun, L. (2010). Analysis on spatial heterogeneity of h1n1 flu based on gis spatial analysis technology. In International Conference on Multimedia Technology (ICMT 2010), pages 1-4.

Jiang, S., Zhang, J., Jin, J., Zhang, D., and Wang, T. (2011). Spatial analysis of influenza a $\mathrm{h} 1 \mathrm{n} 1$ and evaluation of risk factors in changsha city based on gis. In 19th International Conference on Geoinformatics, pages 1-5.

Kandwal, R., Garg, P. K., and Garg, R. D. (2009). Health GIS and hiv/aids studies: perspective and retrospective. Journal of Biomedical Informatics, 42(4):748-755.

Maia, R. S., von Wangenheim, A., and Nobre, L. F. (2006). A statewide telemedicine network for public health in brazil. In IEEE 19th International Symposium on Computer-Based Medical Systems (CBMS 2006), pages 495-500.

Moreira, R. d. S., Nico, L. S., and Tomita, N. E. (2007). A relação entre o espaço e a saúde bucal coletiva: por uma epidemiologia georreferenciada. Ciência e Saúde Coletiva, 12:275 - 284.

Ohannessian, R. (2015). Telemedicine: potential applications in epidemic situations. European Research in Telemedicine / La Recherche Européenne en Télémédecine, 4(3):95-98.

Parker, J., Wei, Y., Yates, A., Frieder, O., and Goharian, N. (2013). A framework for detecting public health trends with twitter. In Proceedings of the 2013 IEEE/ACM International Conference on Advances in Social Networks Analysis and Mining, ASONAM '13, pages 556-563, New York, NY, USA. ACM.

Rasam, A. R. A., Noor, A. M. M., Ahmad, N., and Ghazali, R. (2011). Mygeohealth: Gisbased cholera transmission risk system in sabah, malaysia. In IEEE 7th International Colloquium on Signal Processing and its Applications (CSPA 2011), pages 474-479.

Rede Interagencial de Informação para a Saúde (2008). Indicadores básicos para a saúde no Brasil: conceitos e aplicações. Organização Pan-Americana da Saúde.

Sauro, J. and Lewis, J. R. (2011). When designing usability questionnaires, does it hurt to be positive? In Proceedings of the SIGCHI Conference on Human Factors in Computing Systems, CHI '11, pages 2215-2224, New York, NY, USA. ACM.

Shi, H., Zhang, Y., Zhang, J., Wan, P., and Shaw, K. (2007). Development of webbased epidemiological reporting system for tasmania utilizing a google maps add-on. In Digital Image Computing Techniques and Applications, 9th Biennial Conference of the Australian Pattern Recognition Society on, pages 118-123.

Widener, M. J. and Li, W. (2014). Using geolocated twitter data to monitor the prevalence of healthy and unhealthy food references across the US. Applied Geography, 54:189197.

World Health Organization (2010). Telemedicine: opportunities and developments in member states: report on the second global survey on ehealth 2009. http: / /www . who.int/goe/publications/goe_telemedicine_2010.pdf. 